DOI: 10.2478/adms-2021-0002

\author{
R. Maharudra* ${ }^{* 1,3}$, T. Rajanna ${ }^{2}$, B. Arya ${ }^{1}$ \\ ${ }^{1}$ Department of Mechanical Engineering, B.M.S College of Engineering , Bengaluru-560019, \\ Karnataka, India \\ ${ }^{2}$ Department of Civil Engineering, B.M.S College of Engineering , Bengaluru-560019, Karnataka, \\ India \\ ${ }^{3}$ Department of Mechanical Engineering, Government Polytechnic College , Bagepalli-561207, \\ Karnataka, India \\ *rudresh2100@gmail.com
}

\title{
EFFECT OF TRAPEZOIDAL SHAPES ON THE THERMAL BUCKLING BEHAVIOUR OF PERFORATED COMPOSITE PLATES
}

\begin{abstract}
Thermal buckling study on the symmetric laminated composite trapezoidal plate with a circular cutout subjected to a uniform increase in temperature for various boundary conditions is explored in this paper. In a mathematical model, the first-order shear deformation principle is employed in accordance with the variational energy system. For acquiring the thermal buckling temperature, a nine-node heterosis plate relation has been used in the finite element formulation. By correlating the present findings with accessible literature, the effectiveness of the present formulation is verified. The impact of different parameters, such as trapezoidal shape, cutout size, ply-orientation, plate edge conditions and plate width to thickness ratio have been considered to study the effect of each parameters on the buckling characteristics of plate under various temperatures. It is observed from the study that each parametric investigation significantly affect the thermal buckling behaviour of trapezoidal plates.
\end{abstract}

Keywords: Trapezoidal shape; cutout offset; laminated composite; heterosis element; thermal load

\section{INTRODUCTION}

Advances in material science have resulted in a remarkable rise in the allowable material stress level. It is more evident in the case of light-weight and high-strength fibre-reinforced composite materials. These materials are extensively used in laminated thin-walled structural parts for various modern engineering structures in mechanical, civil and aerospace engineering sectors. The diverse usage and application of such thin walled laminated composites which are highly susceptible to buckle at low stress levels has been the subject of interest for researchers owing to its importance in the design application of the structures. In most of the cases, these laminated 
composites are plate like-structural elements and which are of different shapes. For instance, the aeroplane wings and ship bodies are made up of different shapes of structural elements, namely square, rectangular, trapezium, etc. Most of the literature covers the stability analysis of rectangular and square plates under mechanical and thermal loads, but trapezoidal plates are also commonly found. And there is not enough literature regarding these trapezoidal plates. Further, in many situations, these trapezoidal plates are provided with cutouts or openings in order to facilitate many functional requirements, such as easy access for inspection, providing hydraulic lines, fuel lines and electrical lines, often simply reducing the structural total weight [1]. Nevertheless, during the initial fabrication and final use, the laminated composites are subjected to changing environmental conditions. Out of various environmental loads that may exist, the ones that are of interest to us arise from two considerations. The first one is the effect of temperature known as thermal effects [2]. The second one is the effect of absorption of moisture from the atmosphere known as hygroscopic effects. The in-plane thermal stress distribution within the plate may be more complicated by the shape of the trapezium and discontinuities, thereby the making stress field is highly non-uniform in nature, resulting in the loss of stability at an extremely low levels of stress. Therefore, a complete knowledge of thermal buckling behaviour of trapezium plates with cutouts is most necessary in order to prevent the premature failure and also to use their full strength. This can be explored to a great extent in the area of thin walled structures. Since the shapes of fibrereinforced composites are highly vulnerable to elevated temperature during the operational life, these are considered as the subject of interest for the present work.

Manickam et al. [3] and Biswal et al. [4] studied the effect of hygrothermal loading on the buckling behaviour of laminated composite plates by adopting the finite element technique (FE). A quadratic iso-parametric eight-noded element with first-order shear deformation theory has been used in their study. This study is restricted to square/rectangular plates without any openings. However, for certain mesh configuration and boundary conditions, the eight-noded plate element appears to be locks in shear, even when selective or reduced integration techniques are used for a thin plate configuration. It is also stated that the eight-node serendipity element is comparatively more sensitive to element aspect ratios and element shape distortions Palani et al. [5]. Numerical investigation based on the finite element technique (FE) were performed by Panda et al. [6] to study the hygrothermal effect on the free vibration of delaminated woven composite plates. Behera et al. [7] investigated the effect of variable thermal environment on the free vibration, stability and flexural behaviour of clamped composite laminated plates without cutouts by using commercial software ABAQUS. Jeyaraj [8] examined the effect of varying temperature distributions on the free vibration and buckling behaviour of an isotropic plate by using FE technique. Ram and Sinha [9] have investigated the effect of hygrothermal environment on the buckling behaviour of laminated plates by using FE technique. The effect of thickness, boundary conditions and different elevated temperature as well as moisture has been considered in their study to investigate the buckling characteristics of symmetric and anti-symmetric laminated plate. Twinkle et al. [10] carried out numerical and experimental investigation to study the influence of local heating on the vibration analysis of an isotropic plate. It has been revealed in their study that the elevated temperature significantly affects the vibration modes of the plate. Also the free vibration of the plate predominantly decreases with the increase in the elevated temperature. Yang et al. [11] investigated the effect of pressure load on the thermal buckling and dynamic characteristics of composite plates by using FE technique. They showed in their study that the pressure distribution has a significant effect on the buckling mode shapes of the plate. The hygro-mechanical coupling and multiscale swelling coefficients evaluation of flax yarns and flax/epoxy composites have 
been investigated by Abida et al. [12]. Hygro-thermal effect on the tapered laminated composite structural members was conducted by Gayen and Roy [13], in which they utilized an analytical approach to find out stress distributions such as axial in-plane and inter-laminar shear stresses in their study. Biswal [14] investigated the effect of hygro-thermal impact on the buckling behaviour of laminated composite cylindrical shells by conducting experiment as well as numerical investigation. Patel et al. [15] utilized higher-order concepts to do his research on hygro-thermal effect on the structural behaviour of thick composite laminates. The effect of variation in temperature on the buckling behaviour of hybrid angle-ply laminated composite plate with a cutout was studied by Sahin [16]. Chikh et al. [17] contributed their research on the thermal buckling study using a simplified HSDT for cross-ply laminated plates. Chandrashekhara [18] has investigated the effect of temperature on buckling behaviour of laminated plates using a deformation flexible finite element formulation. Hsu and Sun [19] analyzed the effect of thermal buckling by using the theory of transverse shear deformation of composite laminates. By using a finite element approach, Chockalingam et al. [20] evaluated the effect of critical temperature on the hybrid laminates. An impact of temperature on the buckling action of composite laminated plates has been observed by Thangaratnam and Ramachandran [21]. The effect of thermally induced buckling behaviour of thicker composite laminated plate in the use of non-uniform temperature concentration has been reported by Chen et al. [22]. The outcomes of variations in moisture and temperature on the frequency responses of skew laminated interbred composite and sandwich plates have been achieved by Kattimani et al. [23]. Nguyen-Xuan et al. [24-26] presented the study on the generalized shear deformation theory for static, dynamic and buckling analysis of functionally graded materials (FGM) and sandwich plates.

From this brief overview of the past literature, it has been observed that a vast amount of work deals with the vibration and buckling behaviour of laminated square and rectangular plates exposed to hygrothermal loads. On the contrary, relatively less amount of research work deals with the effect of thermal loads with centrally located circular cutouts, but they are restricted to square/rectangular laminated plates only. There is very scanty literature related to the buckling behaviour of trapezoidal plates with and without cutouts under thermal loads. To the best knowledge of the authors, there is no work identified in the literature which relates to the effect of thermal loads and circular cutouts on the buckling characteristics of trapezoidal laminates. Further, it is also observed from the available literature that in finite element formulation, most of the authors have used eight-noded serendipity elements by considering the effect of shear deformation. However, the eight-noded serendipity element is comparatively more sensitive to element aspect ratios and element shape distortions as compared to that of nine-noded Lagrangian elements. Even though the nine-noded Lagrangian element exactly interpolates the quadratic displacement fields and has nearly optimal performance, the stiffness matrix exhibits rank deficiency resulting in the appearance of spurious mechanisms, i.e. zero energy modes. These communicable mechanisms may result in erratic solutions. These shortcomings, namely locking and spurious mechanisms can be completely eliminated by developing a nine-noded heterosis element. This element exhibits improved characteristics as compared to each of the previous quadratic elements and offers high accuracy for extremely thin plate configuration [27-29]. Therefore, by taking into account various parameters such as trapezoidal shape, cutout offset, ply-orientation, plate edge condition and width to thickness ratio, an effort is made to study the impact of trapezoidal shape and the cutout offsets on the thermal buckling behaviour of trapezoidal plate through this work by adopting nine-noded heterosis element. 


\section{MATHEMATICAL FORMULATION}

The basic form of the problem considered here is a trapezoidal shaped plate with a circular cutout, whose geometry and meshing pattern considered are shown in Figs 1(a) and 1(b) respectively.

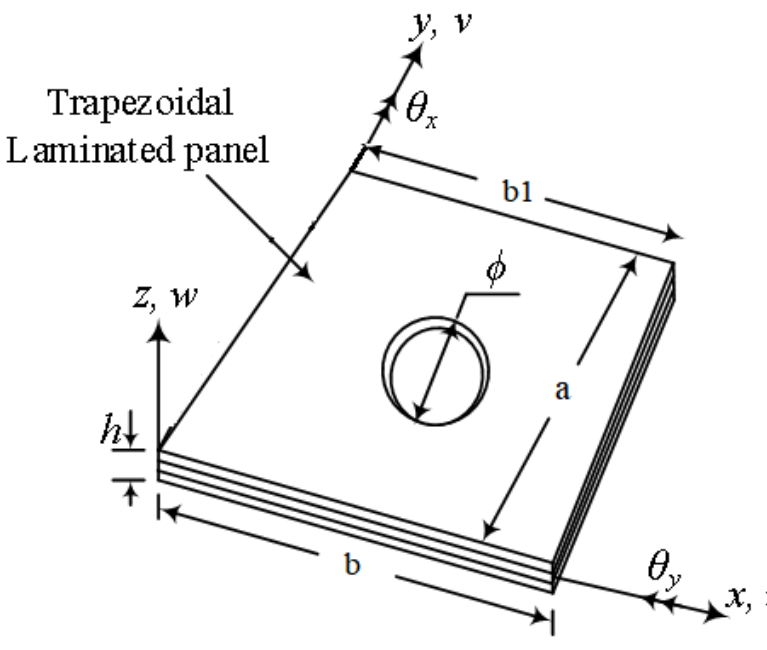

(a)

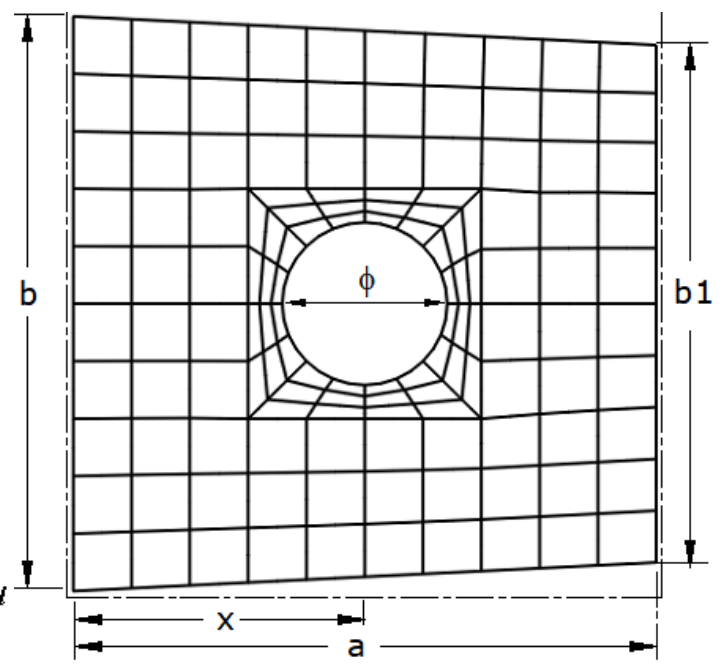

(b)

Fig. 1. (a) Geometry of the plate with its co-ordinate system. (b) Detailed mesh pattern

The trapezoidal laminated plate consists of $N$ layers, each layer is of thickness $t_{n}$, and total thickness $h$, so that $h=\sum_{n=1}^{N} t_{n}$. The laminate lateral measurements are $b$ and $b_{1}$ and longitudinal measurement $a$ are exposed to a homogeneous temperature variation $\Delta T$ between laminated plate and ambient. Here $x$ denotes cutout offset distance from the origin and $\phi$ denotes cutout diameter as shown in Fig. 1(b). The linear stress-strain relationship for each layer is expressed with $x, y$ axes and has the form:

$$
\begin{aligned}
& \left\{\begin{array}{c}
\sigma_{x x} \\
\sigma_{y y} \\
\tau_{x y}
\end{array}\right\}_{n}=\left[\begin{array}{lll}
\overline{\bar{Q}}_{11} & \overline{\bar{Q}}_{12} & \overline{\bar{Q}}_{16} \\
\overline{\bar{Q}}_{12} & \overline{\bar{Q}}_{22} & \overline{\bar{Q}}_{26} \\
\overline{\bar{Q}}_{16} & \overline{\bar{Q}}_{26} & \overline{\bar{Q}}_{66}
\end{array}\right]_{n}\left\{\begin{array}{l}
\varepsilon_{x x}-\alpha_{x x} \Delta T \\
\varepsilon_{y y}-\alpha_{y y} \Delta T \\
\gamma_{x y}-\alpha_{x y} \Delta T
\end{array}\right\}_{n}, \\
& \left\{\begin{array}{c}
\tau_{y z} \\
\tau_{x z}
\end{array}\right\}=\left[\begin{array}{ll}
\overline{\bar{Q}}_{44} & \overline{\bar{Q}}_{45} \\
\overline{\bar{Q}}_{45} & \overline{\bar{Q}}_{55}
\end{array}\right]\left\{\begin{array}{l}
\gamma_{y z} \\
\gamma_{x z}
\end{array}\right\},
\end{aligned}
$$

where $\sigma_{x x}, \sigma_{y y}, \tau_{x y}, \tau_{y z}$ and $\tau_{x z}$ are the stress components, $\Delta T$ is the temperature raise, $\alpha_{x x}$, $\alpha_{y y}$ are the thermal expansion coefficients in $x$ and $y$ direction respectively, $\alpha_{x y}$ is the coefficient of thermal shear, such as [16]: 


$$
\begin{aligned}
& \alpha_{x x}=\alpha_{1} \cos ^{2} \theta+\alpha_{2} \sin ^{2} \theta, \\
& \alpha_{y y}=\alpha_{2} \cos ^{2} \theta+\alpha_{1} \sin ^{2} \theta, \\
& \alpha_{x y}=2\left(\alpha_{1}-\alpha_{2}\right) \sin \theta \cos \theta .
\end{aligned}
$$

Here $\alpha_{1}$ and $\alpha_{2}$ are the constants of thermal expansion in longitudinal and transverse directions of the fibre, respectively.

The Reissner-Mindlin's plate theory is employed in this study based on the assumption that the mid-plane normal remains straight but not necessarily perpendicular to the axis after deflection. The displacement field are stated as [28]:

$$
\begin{aligned}
& u(x, y, z)=u_{0}(x, y)+z \theta_{x}(x, y), \\
& v(x, y, z)=v_{0}(x, y)+z \theta_{y}(x, y), \\
& w(x, y, z)=w_{0}(x, y),
\end{aligned}
$$

where $\theta_{y}$ and $\theta_{x}$ are rotations of the mid-plane with respect to $y$ and $x$ axes respectively and $u_{0}$, $v_{0}$ and $w_{0}$ are the displacements an arbitrary point $(x, y)$ on the mid-plane.

The bending strains $\varepsilon_{x x}, \varepsilon_{y y}$ and shear strains $\gamma_{x y}, \gamma_{y z}, \gamma_{x}$ at any point of the laminate are:

$$
\begin{gathered}
\left\{\begin{array}{l}
\varepsilon_{x x} \\
\varepsilon_{y y} \\
\gamma_{x y}
\end{array}\right\}=\left|\begin{array}{c}
\frac{\partial u_{0}}{\partial x} \\
\frac{\partial v_{0}}{\partial y} \\
\frac{\partial u_{0}}{\partial y}+\frac{\partial v_{0}}{\partial x}
\end{array}\right|+z\left|\begin{array}{c}
\frac{\partial \theta_{x 0}}{\partial x} \\
\frac{\partial \theta_{y}}{\partial y} \\
\frac{\partial \theta_{x}}{\partial y}+\frac{\partial \theta_{y}}{\partial x}
\end{array}\right| \\
\left|\begin{array}{l}
\gamma_{y z} \mid \\
\gamma_{x z}
\end{array}\right|=\left|\begin{array}{l}
\frac{\partial w_{0}}{\partial y}+\theta y \\
\frac{\partial w_{0}}{\partial y}+\theta x
\end{array}\right| .
\end{gathered}
$$

The resultant forces $N_{x x}, N_{y y}$ and $N_{x y}$, moments $M_{x x}, M_{y y}$ and $M_{x y}$ and shearing forces $Q_{x x}, Q_{y y}$ per unit length of the plate are given as

$$
\begin{aligned}
& {\left[\begin{array}{ll}
N_{x x} & M_{x x} \\
N_{y y} & M_{y y} \\
N_{x y} & M_{x y}
\end{array}\right]=\int_{-h / 2}^{h / 2}\left\{\begin{array}{l}
\sigma_{x x} \\
\sigma_{y y} \\
\tau_{x y}
\end{array}\right\}(1, z) d z} \\
& \left\{\begin{array}{l}
Q_{x x} \\
Q_{y y}
\end{array}\right\}=\int_{-h / 2}^{h / 2}\left\{\begin{array}{l}
\tau_{x z} \\
\tau_{y z}
\end{array}\right\} d z
\end{aligned}
$$


The thermal load total potential energy $\pi$ of a laminated plate is equated to:

$$
\pi=U_{b s}+U_{s s}+\Gamma
$$

where $U_{b s}, U_{s s}$ and $\Gamma$ denotes the strain energy of bending, strain energy of shear and potential energy, respectively of in-plane loadings due to the alteration in temperature:

$$
\begin{aligned}
& U_{b s}=1 / 2 \int_{-h / 2}^{h / 2}\left[\iint_{r}\left(\sigma_{x} \varepsilon_{x x}+\sigma_{y} \varepsilon_{y y}+\tau_{x y} \gamma_{x y}\right) d \Lambda\right] d z, \\
& U_{s s}=1 / 2 \int_{-h / 2}^{h / 2}\left[\iint_{r}\left(\tau_{x z} \gamma_{x z}+\tau_{y z} \gamma_{y z}\right) d \Lambda\right] d z, \\
& \Gamma=1 / 2 \iint_{r}\left[\bar{N}_{1}\left(\partial w_{0} / \partial x\right)^{2}+\bar{N}_{2}\left(\partial w_{0} / \partial y\right)^{2}\right. \\
& \left.+2 \overline{N_{12}}(\partial w / \partial x)(\partial w / \partial y)\right] d \Lambda-\int_{\partial r}\left(\bar{N}_{n}^{b} u_{n}^{0}+\bar{N}_{s}^{b} u_{s}^{0}\right) d s,
\end{aligned}
$$

where $d \Lambda=d x d y, r$ is the plate region excluding hole. $\bar{N}_{n}^{b}, \bar{N}_{s}^{b}$ are in-plane loads applied on the boundary $\partial r$.

To achieve equilibrium, the potential energy $\pi$ must be stationary. The equilibrium equations of the angle-ply laminated plate subjected to temperature change can be derived from the variational principle through the use of stress-strain and strain-displacement relations. One may obtain these equations by $\partial \pi=0$ [27].

\section{Finite element formulation}

A computer program by using FORTRAN language has been developed to work out buckling problems. The different participating structural matrices are obtained by assembling each individual element level matrices by using skyline technique. Eigenvalues and Eigenvectors involved are obtained by using subspace iteration technique [27]. In the computer program, selective integration scheme, in which $3 \times 3$ gauss rule for in plane as well as bending terms and $2 \times 2$ gauss rule for shear terms has been incorporated to generate element elastic stiffness matrix without any shear locking. Similarly, a full integration scheme (i.e., 3 x 3 gauss rule) has been used for both geometric stiffness as well as mass matrices. Fig. 2 shows the schematic example of various types of elements. The 9-noded heterosis element as shown in Fig. 2 has been used in the discretization of the trapezoidal plate. 


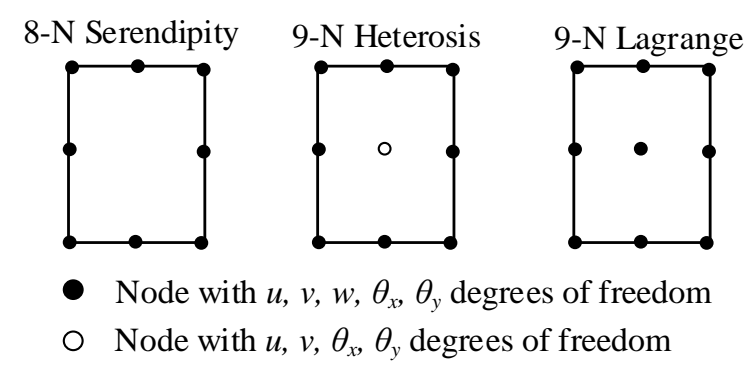

Fig. 2. Schematic example of various types of elements

The 9-noded heterosis element is generated by incorporating 9-noded Lagrangian shape functions for $u, v, \theta_{x}, \theta_{y}$ and 8-noded serendipity shape functions for $w$ degrees of freedom. A 9noded heterosis plate element has been employed in the current research to study the buckling behaviour of the trapezoidal plate. This type of element exhibits high level accuracy as compared to that of 8-noded serendipity and 9-noded Lagrangian elements [29].

By the use of minimum potential energy rule, the stiffness matrix of the plate is achieved. Bending stiffness $\left[K_{b s}\right]$, shear stiffness $\left[K_{s s}\right]$ and geometric stiffness $\left[K_{g s}\right]$ can be represented as:

$$
\begin{aligned}
& {\left[K_{b s}\right]=\int_{\Lambda}\left[B_{b s}\right]^{T}\left[D_{b s}\right]\left[B_{b s}\right] d \Lambda,} \\
& {\left[K_{s s}\right]=\int_{\Lambda}\left[B_{s s}\right]^{T}\left[D_{s s}\right]\left[B_{s s}\right] d \Lambda,}
\end{aligned}
$$

and

$$
\left[K_{g s}\right]=\int_{\Lambda}\left[B_{g s}\right]^{T}\left[D_{g s} \llbracket B_{g s}\right] d \Lambda
$$

where,

$$
\begin{gathered}
{\left[D_{b s}\right]=\left[\begin{array}{ll}
A_{i j} & B_{i j} \\
B_{i j} & D_{i j}
\end{array}\right],} \\
{\left[D_{s s}\right]=\left[\begin{array}{cc}
k_{1}^{*} A_{44} & 0 \\
0 & k_{2}^{*} A_{45}
\end{array}\right],} \\
{\left[D_{g s}\right]=\left[\begin{array}{cc}
\bar{N}_{1} & \bar{N}_{12} \\
\bar{N}_{12} & \bar{N}_{2}
\end{array}\right]} \\
\left(A_{i j}, B_{i j}, D_{i j}\right)=\int_{-h / 2}^{h / 2} \overline{\bar{Q}}_{i j}\left(1, z, z^{2}\right) d z \quad(i, j=1,2,6), \\
\left(A_{44}, A_{55}\right)=\int_{-h / 2}^{h / 2}\left(\overline{\bar{Q}}_{44}, \overline{\bar{Q}}_{45}\right) d z .
\end{gathered}
$$


Here $\left\lfloor A_{i j}\right\rfloor,\left\lfloor B_{i j}\right\rfloor$ and $\left\lfloor D_{i j}\right\rfloor$ represents extension, extension-bending and bending stiffness respectively and $k_{1}^{*}=k_{2}^{*}=5 / 6$ represents the shear correction factors.

\section{Governing Equations}

After assembling the elastic stiffness and the geometric stiffness matrices, the corresponding eigenvalue problem can be solved by using any standard extraction procedures [30].

$$
\left.\llbracket \bar{K}_{0}\right]-\lambda_{c r}\left[\bar{K}_{0 g} \rrbracket\left\{\begin{array}{l}
u_{i} \\
v_{i} \\
w_{i}
\end{array}\right\}=0\right.
$$

where

$$
\begin{aligned}
& {\left[\bar{K}_{0}\right]=\left[K_{b s}\right]+\left[K_{s s}\right]} \\
& -\lambda_{c r}\left[\bar{K}_{0 g}\right]=\left[K_{g s}\right]
\end{aligned}
$$

The critical buckling temperature $T_{c r}$ is that which corresponds to the smallest eigen-value $\lambda_{c r}$ is equals to

$$
\begin{gathered}
T_{c r}=\lambda_{c r} \\
\Delta T=T_{f}-T_{r}
\end{gathered}
$$

where, $\Delta T$ is the temperature difference between the final temperature $\left(T_{f}\right)$ and the reference temperature $\left(T_{r}\right)$.

\section{RESULTS AND DISCUSSION}

There are several approaches for solving eigenvalue study. In this analysis, the subspace iteration technique has been adopted to solve the eigenvalues problem. The angle-ply and crossply laminated trapezoidal composite plates with an eccentrically placed circular cutout considered and are symmetrically bonded.

The trapezoidal plate considered in this research is thin in nature with $h / b=0.01$, having symmetrical 4-layers unless otherwise stated. The thermo-elastic properties of the material that are used for E-glass / epoxy are shown in Table 1. 
Table 1. Material properties used for numerical solutions at different temperature [9]

\begin{tabular}{ccccccc}
\hline \multirow{2}{*}{$\begin{array}{c}\text { Elastic Moduli } \\
(\mathrm{GPa})\end{array}$} & 300 & 325 & 350 & 375 & 400 & 425 \\
\cline { 2 - 7 } & 130.0 & 130.0 & 130.0 & 130.0 & 130.0 & 130.0 \\
\hline$E_{11}$ & 9.50 & 8.50 & 8.00 & 7.50 & 7.00 & 6.75 \\
$E_{22}$ & 6.00 & 6.00 & 5.50 & 5.00 & 4.75 & 4.50 \\
$G_{12}$ & & & & & & \\
\hline
\end{tabular}

$G_{12}=G_{13}, G_{23}=0.5 G_{13}, \mu_{12}=0.3, \alpha_{1}=-0.3 \mathrm{E}^{-6} / \mathrm{K}$ and $\alpha_{2}=28.1 \mathrm{E}^{-6} / \mathrm{K}$ here $E_{11}, E_{22}$, and $\mu_{12}$ are modulus of elasticity in the direction of 1 and 2 and Poison's ratio respectively, $\alpha_{1}$ and $\alpha_{2}$ are the coefficients of thermal expansion of the materials considered in the solution.

In the string of any support conditions, the notations SSSS and CCCC indicate simply support and clamped boundary conditions respectively. The boundary conditions adopted in this work are as follows [31]:

(1) Simply supported condition (SSSS):

$$
\begin{aligned}
& \text { At } x=0, a ; u_{0}=w_{0}=\theta_{y}=0 \\
& \text { At } y=0, b ; v_{0}=w_{0}=\theta_{x}=0
\end{aligned}
$$

(2) Clamped condition (CCCC):

$$
\text { At } x=0 \text {, } a \text {; and } y=0, b ; u_{0}=v_{0}=w_{0}=\theta_{x}=\theta_{y}=0
$$

\section{Convergence and comparison study}

The convergence study is most necessary in order to decide the size of the mesh to be considered to get moderately good converged results. In this study, a 10 x 10 mesh size is found to be optimally converged and hence, the same mesh size is chosen throughout this work. On the other hand, a comparison study is also most essential in order to decide the accuracy of the present finite element formulation developed to carry out the study of the thermal buckling problems. The effect of aspect ratios of plate on the thermal buckling has been studied and the numerical results are shown in Table 2 along with the results of Chandrashekhara [18]. It is observed that the present results agree with the literature. 
Table 2. Comparison of the temperature buckling loads of simply supported (SSSS) isotropic plate. $E=1.0 \times 10^{9} \mathrm{~Pa}, \mu=0.3, a / \mathrm{h}=100, a / b=1, a=254, \alpha_{0}=1.0 \mathrm{E}^{-6}$

\begin{tabular}{|c|c|c|c|c|c|c|}
\hline \multirow{4}{*}{$\mathrm{a} / \mathrm{b}$} & \multicolumn{5}{|c|}{ Critical thermal buckling $\left(\alpha \mathrm{T}_{\mathrm{cr}} \times 10^{-4}\right)$} & \multirow{3}{*}{$\begin{array}{c}\text { Chandrashekhara [18] } \\
\text { Mesh size }\end{array}$} \\
\hline & \multicolumn{5}{|c|}{ Present } & \\
\hline & \multicolumn{5}{|c|}{ Mesh size } & \\
\hline & $4 \times 4$ & $5 \times 5$ & $10 \times 10$ & $15 \times 15$ & $20 \times 20$ & $4 \times 4$ \\
\hline 0.25 & 0.6731 & 0.6725 & 0.6720 & 0.6720 & 0.6720 & 0.6727 \\
\hline 0.50 & 0.7918 & 0.7912 & 0.7906 & 0.7905 & 0.7905 & 0.7913 \\
\hline 0.75 & 0.9897 & 0.9889 & 0.9882 & 0.9881 & 0.9881 & 0.9890 \\
\hline 1.00 & 1.2667 & 1.2657 & 1.2648 & 1.2647 & 1.2646 & 1.2657 \\
\hline 1.25 & 1.6227 & 1.6214 & 1.6202 & 1.6201 & 1.6201 & 1.6234 \\
\hline 1.50 & 2.0577 & 2.056 & 2.0546 & 2.0544 & 2.0543 & 2.0561 \\
\hline 1.75 & 2.5715 & 2.5695 & 2.5676 & 2.5674 & 2.5674 & 2.5696 \\
\hline 2.00 & 3.1641 & 3.1615 & 3.1593 & 3.1590 & 3.1589 & 3.1617 \\
\hline 2.25 & 3.8354 & 3.8322 & 3.8295 & 3.8292 & 3.8291 & 3.8324 \\
\hline 2.50 & 4.5850 & 4.5813 & 4.5780 & 4.5777 & 4.5776 & 4.5817 \\
\hline 2.75 & 5.4132 & 5.4088 & 5.4050 & 5.4046 & 5.4044 & 5.4096 \\
\hline 3.00 & 6.3193 & 6.3152 & 6.3098 & 6.3093 & 6.3091 & 6.3144 \\
\hline
\end{tabular}

\section{Case studies on buckling behaviour of trapezoidal plate under uniform thermal loads}

In this section, the buckling action of angle-ply and cross-ply trapezoidal laminates is examined by considering various cutout offsets. The term trapezoidal indicates when the percentage of $b_{1}$ value changes from 0.5 to 1 . For instance, if $b_{1} / b$ ratio equals 1 , it indicates a square plate. Six different temperature combinations have been considered and the performance of each combination on different parametric conditions is investigated in detail in the following sub-sections.

\section{Thermal Buckling behaviour under different ply-orientations}

The effect of thermal buckling performance of trapezoidal plate has been studied by considering trapezoidal shape ratio $\left(b_{1} / b\right) 0.9$ and results are shown in Fig. 3. The cutout offset ratio $(x / a)$ is taken as 0.4 under the action of various temperature. The plate considered here is trapezoidal and all four sides are simply supported. It is evident from Figs 3(a) and 3(b) that the thermal buckling resistance $\left(T_{c r}\right)$ increases with the increase in ply-angle and found to be the highest at a ply-orientation of $( \pm 45)_{s}$ thereafter reduces with further increase in the ply-angle. It is necessary to note that even though the thermal buckling behaviour remains the same for both thin $(b / h=100)$ and thick $(b / h=5)$ plates, the thick plate shows significantly higher thermal buckling resistance as compared to that of thin plate configuration. It may be due to the effect of high stiffness of the material. It is also important to mention that the critical buckling temperature $\left(T_{c r}\right)$ increases with the decrease in temperature, and is found to be the highest at a temperature of 300 K. 


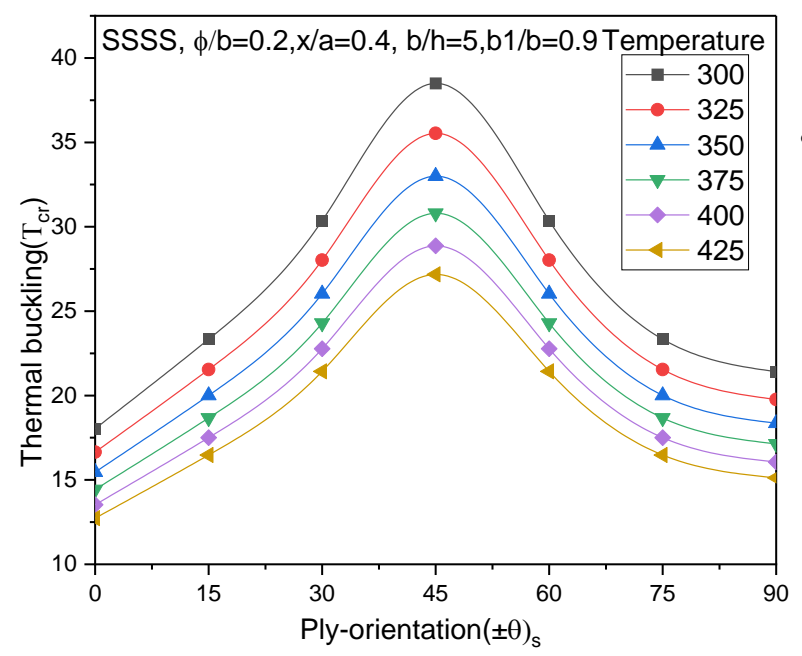

(a)

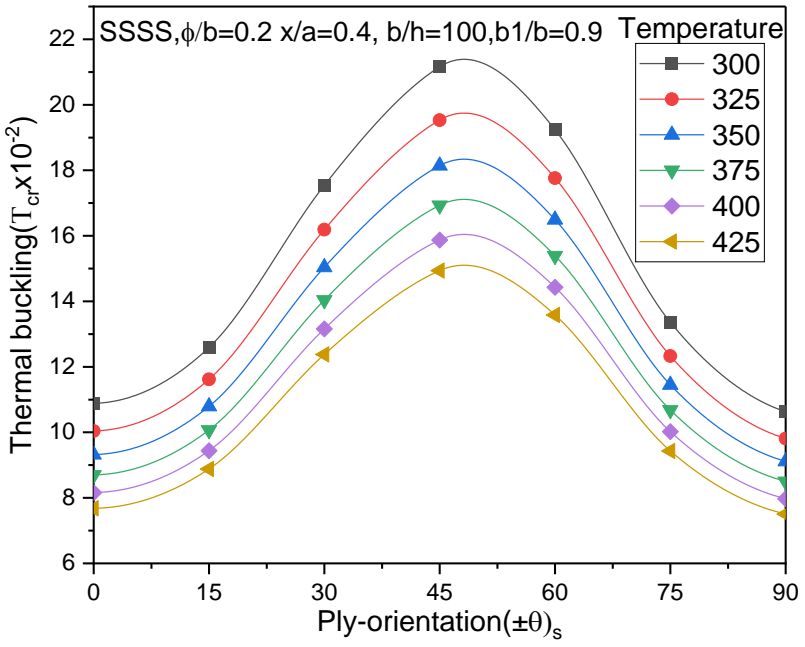

(b)

Fig. 3. Thermal buckling behaviour under (a) thick laminate and (b) thin laminate

\section{Thermal Buckling behaviour under various trapezoidal shape}

An impact of trapezoidal shape ratio on the critical thermal buckling $\left(T_{c r}\right)$ behaviour of crossply laminated plate is observed by considering thick and thin plates and the results are illustrated in Figs 4(a) and 4(b) respectively. The cutout size considered here is medium i.e $\phi b=0.2$ and is located at a distance, $x / a=0.5$. From the Figs 4(a) and 4(b), it is clear that the buckling resistivity of the plate progressively decreases as the temperature increases in both thick and thin laminates. It is also observed that the thermal buckling behaviour of the thick plate is similar to that of thin plate with a significantly higher value of thermal buckling coefficient. Also as the trapezoidal shape ratio increases, irrespective of the $b / h$ ratio, thermal bucking resistance increases and is found to be at its peak when the trapezoidal shape ratio reduces to 0.5 .

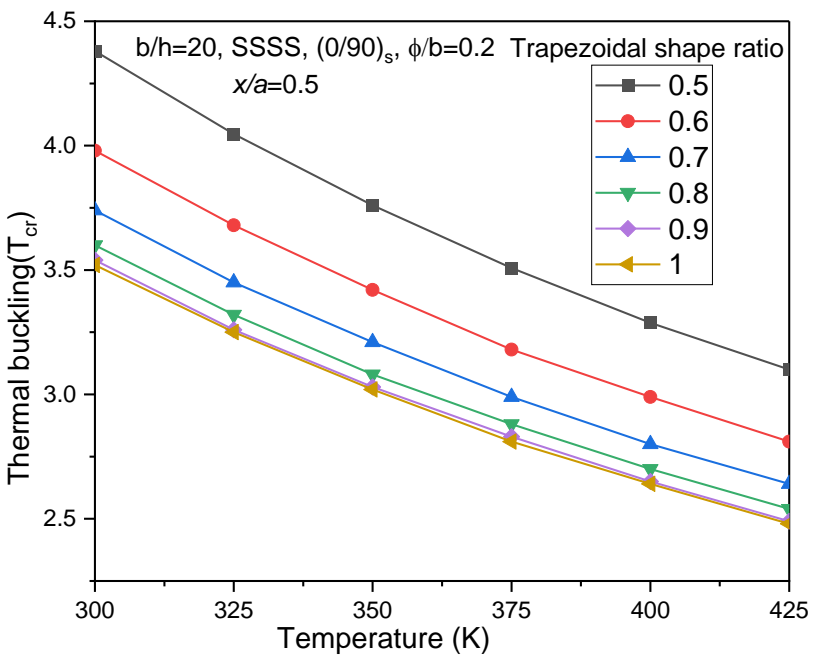

(a)

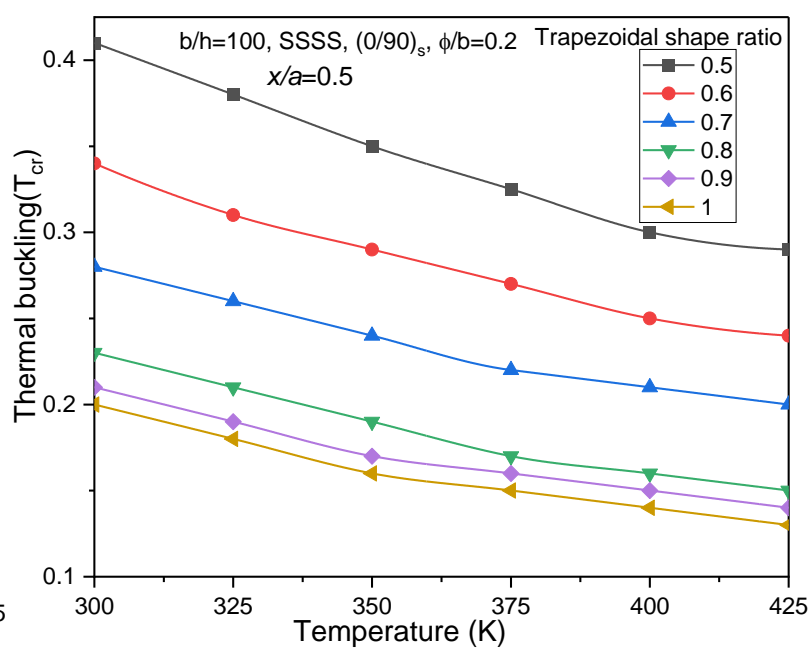

(b)

Fig. 4. Thermal buckling behaviour under different trapezoidal shapes with (a) $b / h=20$ (b) $b / h=100$ 


\section{Impact of cutout offset on Thermal Buckling behaviour}

An impact of cutout offset ratio on the critical thermal buckling $\left(T_{c r}\right)$ activity of trapezoidal configured plate has been observed and the results are shown in Figs 5(a) to 6(b). The cutout offsets considered are $20 \%, 40 \%, 60 \%$ and $80 \%$ of the total length of the plate. Symmetric angle ply orientation of $( \pm 45)_{\mathrm{s}}$ and $(0 / 90) \mathrm{s}$ is considered with cutout size 0.2 under simply supported edge conditions. It is evident from the figures that the thermal buckling stability increases gradually when the cutout moves towards higher stiffness zones. However, this behaviour is completely reversed in thin laminates, and does not show much difference in the buckling load in thin plates as shown in Figs 5 (b) and 6 (b). Therefore, it is clear from this analysis that the thicker laminated plate with $( \pm 45)_{\mathrm{s}}$ has the capability to withstand higher thermal buckling load.

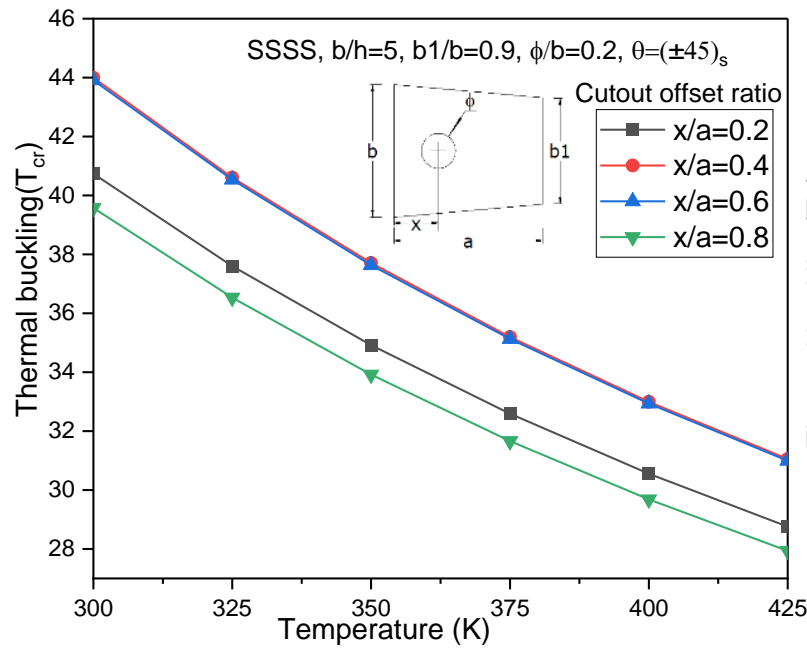

(a)

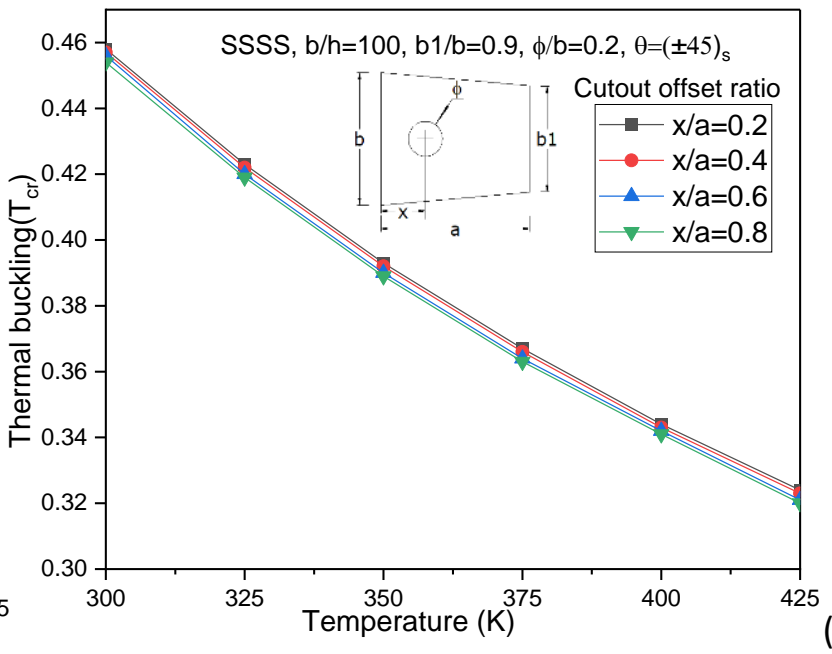

b)

Fig. 5. Thermal buckling behaviour under different cutout offset ratios (a) b/h=5 with $( \pm 45)(b) b / h=100$ with $( \pm 45)$

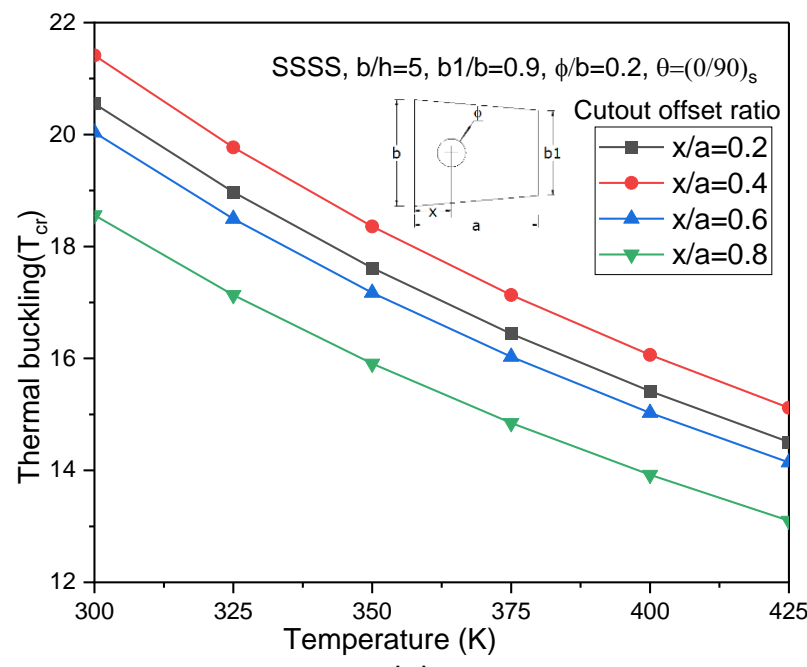

(a)

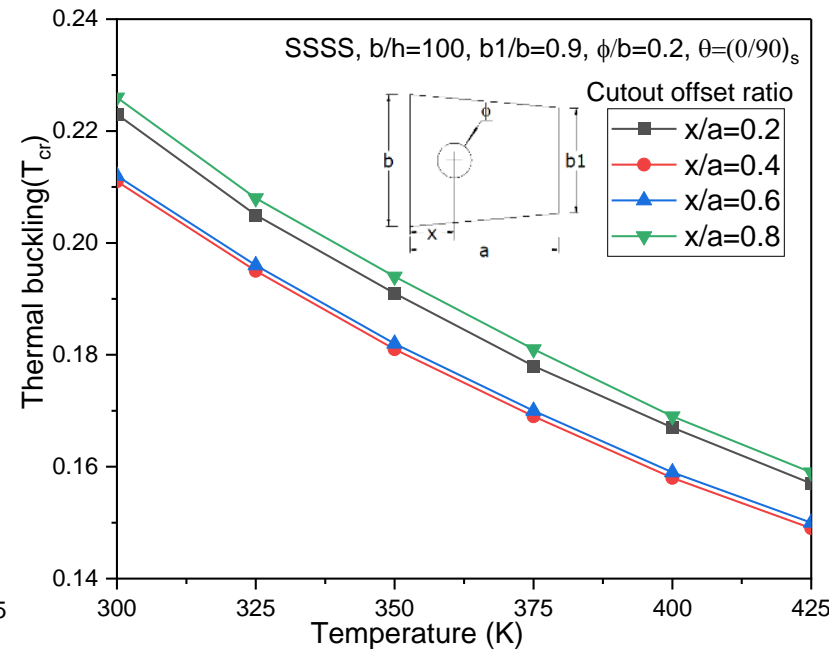

(b)

Fig. 6. Thermal buckling behaviour under different cutout offset ratios where (a) $b / h=5$ and (b) $b / h=100$ 
An impact of different thickness ratio on thermal buckling behaviour

A similar research is carried out to examine the effect of plate thickness ratio by taking into account two different ply configurations with all four edges being simply supported. Trapezoidal shape ratio considered here is 0.9 with cutout ratio $(\phi b)$ is 0.2 . The results are exemplified in Figs 7(a) and 7(b) by considering the cross-ply and angle-ply laminated schemes, respectively. It is very clear from Figs 7(a) and 7(b) that the value of $T_{c r}$ reduces with the increase in plate thickness ratio irrespective of temperature loading. The highest thermal failing load is seen in the case of angle ply laminates i.e $( \pm 45)_{s}$. As the thickness ratio and temperature further increases, there is not much increase in the thermal buckling stability, wherein the quantity of $T_{c r}$ is found to remain almost unchanged for thickness ratios $(b / h)$ as well as temperature.

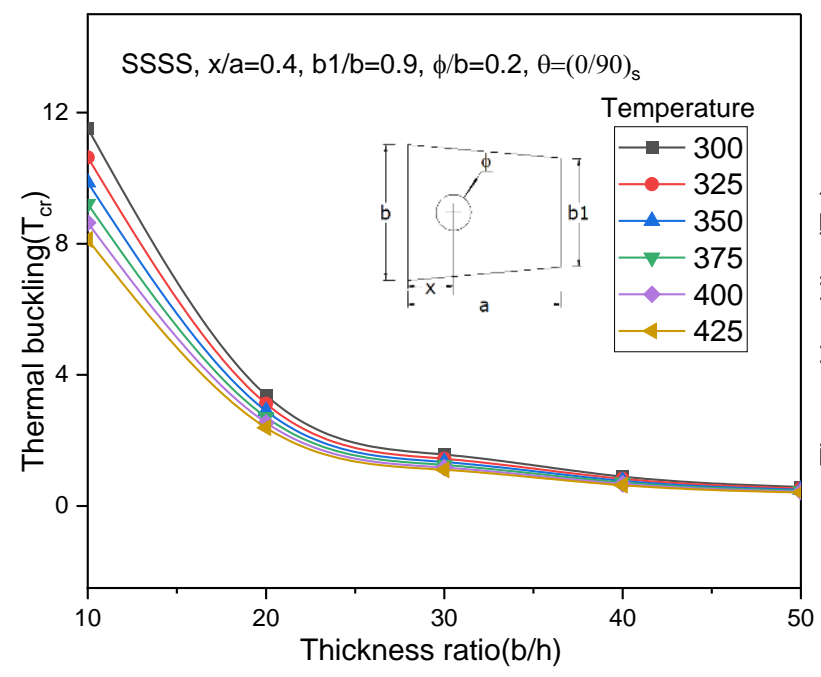

(a)

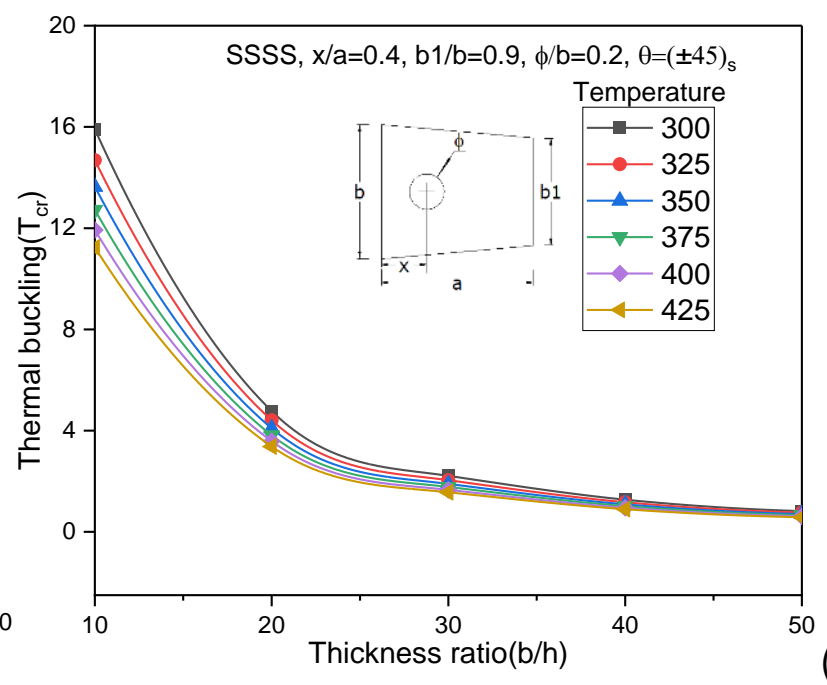

b)

Fig. 7. Thermal buckling behaviour under different thickness (a) cross-ply and (b) angle-ply

\section{Impact of cutout size on thermal buckling parameters of trapezoidal plate}

An impact of cutout size on the thermal buckling behaviour of SSSS and CCCC edged trapezoidal plate has been investigated and the numerical results are shown in Fig. 8 (a) and 8 (b) respectively by considering angle-ply laminated scheme. It is noticed from Figs 8 (a) and 8 (b) that the thermal buckling resistance significantly increases with the increase in the cutout size and follows parabolic variations. The peak thermal buckling load is observed at cutout ratio 0.7 and a temperature of $300 \mathrm{~K}$ and is minimum at a cutout ratio of 0.1 and a temperature of $425 \mathrm{~K}$. As the cutout size increases, the thermal stress distribution within the vicinity of the cutout moves towards the stiffer region of the plate and this may be the reason for the increasing thermal buckling resistance with respect to the cutout size. It is also clear from the study that the CCCC edged plate shows significantly higher buckling resistance as compared with SSSS edge conditioned plate at different temperatures. 


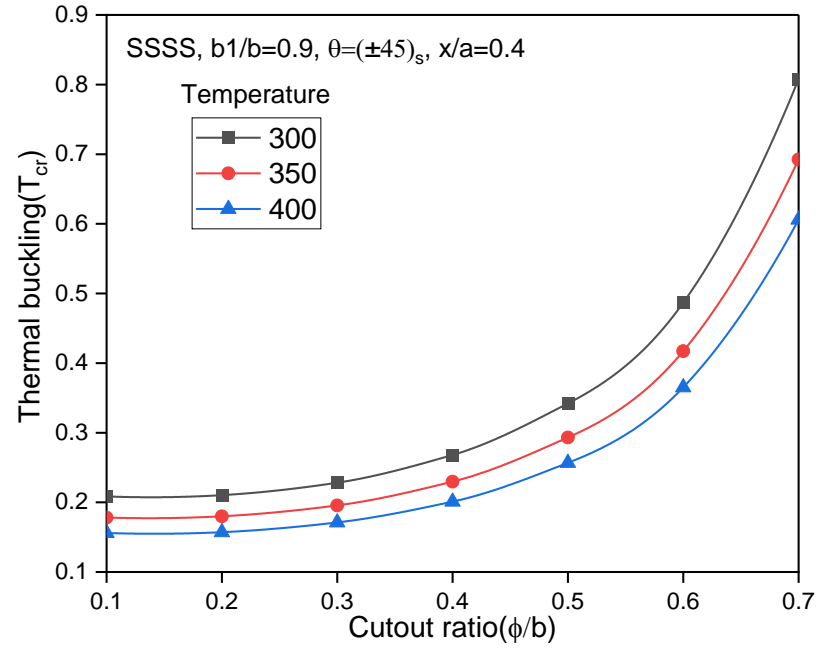

(a)

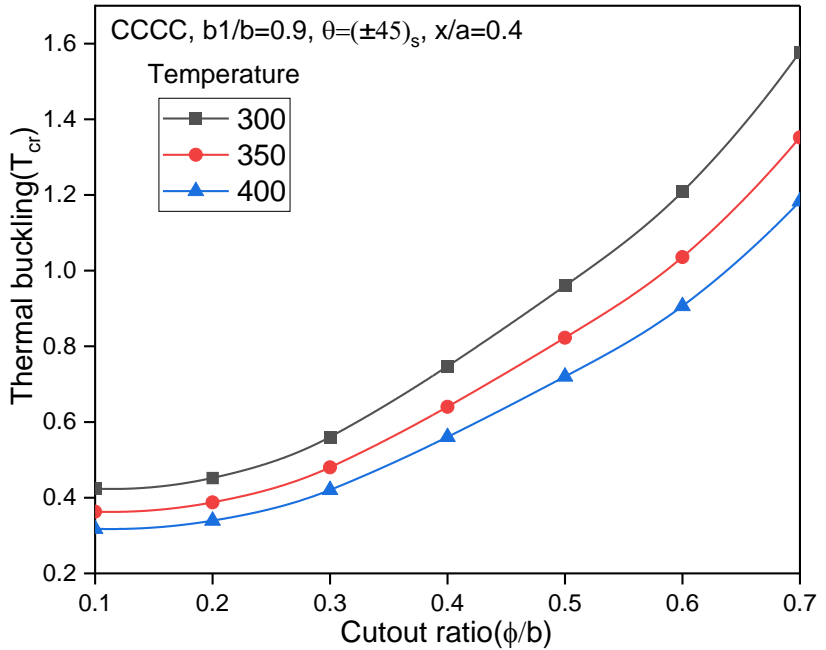

(b)

Fig. 8. Impact of boundary conditions and cutout ratio on the thermal buckling behaviour of (a) SSSS and (b) CCCC edged plates

\section{Impact of Boundary Conditions on trapezoidal plate}

An impact of various boundary conditions on the thermal buckling behaviour of trapezoidal plate has been studied by considering a cutout offset ratio of 0.6 and trapezoidal shape ratio of 0.9 for cross-ply and angle-ply layup schemes and the responses are shown in the Fig. 9(a) and 9(b) respectively. It is observed from Figs 9 (a) and 9 (b) that the buckling resistance of the trapezoidal plate reduces with the increase in temperature and is maximum for CCFF edge condition and minimum for SSFF condition irrespective of plate cutout offset ratios $(x / a)$ as well as temperature. It is also observed from the graph that for any given boundary condition, the value of $T_{c r}$ is initially higher at the temperature of $300 \mathrm{~K}$ and reduces as the temperature increases uniformly.

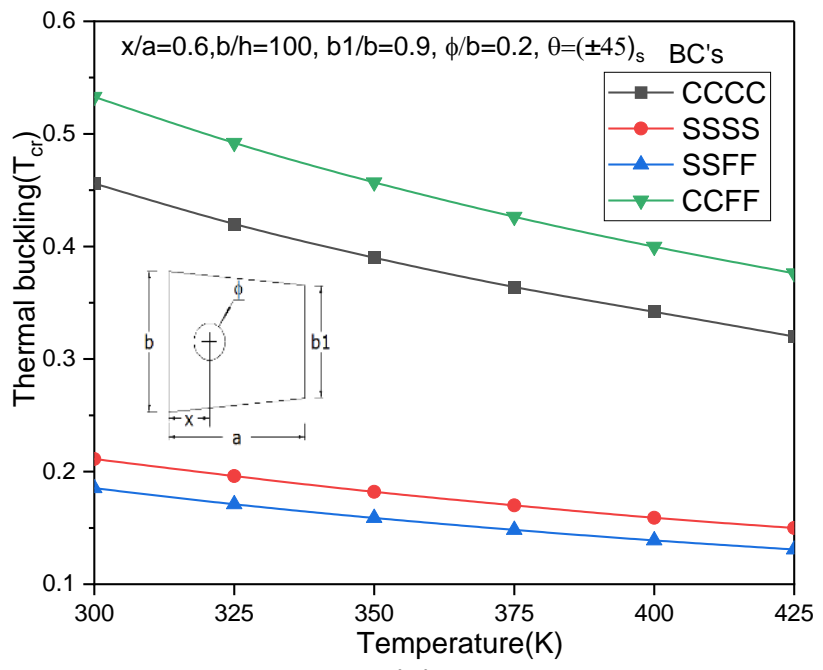

(a)

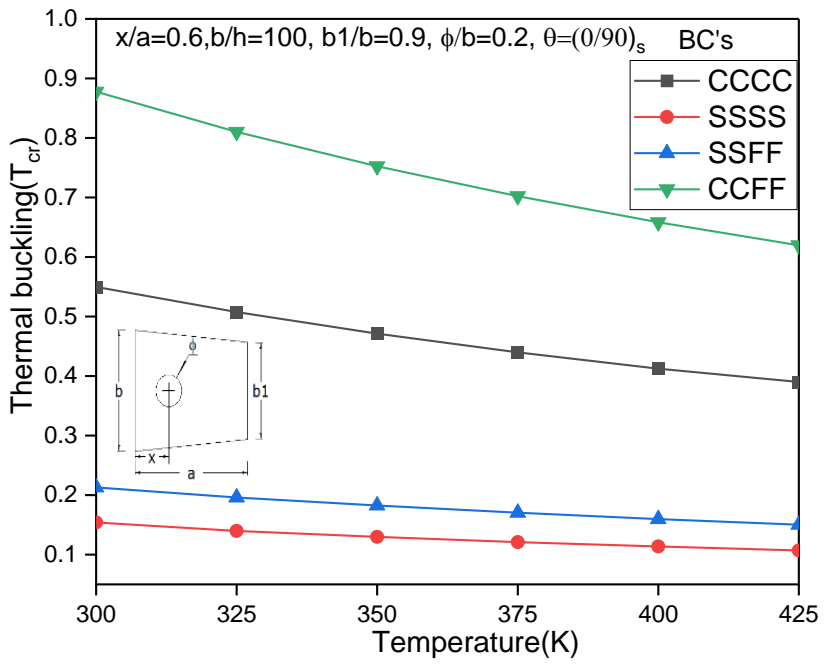

(b)

Fig. 9. Impact of thermal loads on different boundary conditions of (a) angle ply (b) cross ply laminate schemes 


\section{CONCLUSIONS}

Thermal buckling behaviour of trapezoidal plates with different sized cutouts have been studied in this research by considering different intensities of temperature. The important findings from this work are as follows:

- Plate indicates higher buckling temperature as the cutout offset shifts inwards on either edge (left and right). However, the effect of offset is significantly higher for thick plate configuration.

- The critical temperature for buckling depends heavily on the size of the cutout.The existence of a hole initially decreases a laminate's critical thermal load and eventually increases at higher cutout ratios.

- The trapezoidal shape ratio plays a significant role in the thermal buckling resistance. For any given cutout size, the thermal buckling resistance of the plate increases with the increase in trapezoidal shape ratio and found to be highest at $b_{1} / b=0.5$.

- As the thickness ratio increases, the laminated plate becomes thinner in nature, and hence reduces the stiffness of the laminated plate. This reduces the thermal buckling resistance.

- For any given trapezoidal shape as well as temperature, the thermal buckling resistance of the plate decreases with the increase in thickness ratio $(b / h)$. However, the variation in the thermal buckling resistance among different temperatures is significantly higher for thick configuration, but it is insignificant for thin plate configuration.

- For any given trapezoidal shape ratio and temperature, the thermal buckling resistance of the plate increases with the increase in ply-orientation and found to be highest at an angle of $( \pm 45)_{s}$.

- It is also observed from the study that for any given boundary condition, the value of $T_{c r}$ is initially higher at temperature of $300 \mathrm{~K}$ and gradually reduces as the temperature increases uniformly.

\section{REFERENCES}

1. Shiau L.C, Kuo S.Y, Chen C.Y.: Thermal buckling behaviour of composite laminated plates. Composite Structures 92 (2010) 508-514.

2. Mathew T.C, Singh G., Rao G.V.: Thermal buckling of cross-ply composite laminates. Computers \& Structures 42 (2) (1992) 281-287.

3. Manickam G., Bharath A., Das A.N., Chandra A, Barua P.: Thermal buckling behaviour of variable stiffness laminated composite plates. Materials Today Communications 16 (2018) 142-151.

4. Biswal M., Sahu S., Asha A.: Vibration of composite cylindrical shallow shells subjected to hygrothermal loading-experimental and numerical results. Composites Part B: Engineering 98 (2016) 108-119.

5. Palani G.S., Iyer N.R., Rao T.V.R.: An efficient finite element model for static and vibration analysis of eccentrically stiffened plates/shells. Computers \& Structures 43 (4) (1992) 651-661. 
6. Panda H., Sahu S., Parhi P.: Hygrothermal effects on free vibration of delaminated woven fiber composite plates-Numerical and experimental results. Composite Structures 96 (2013) 502-513.

7. Behera R.K., Sharma N., Parida S.K.: Finite element analysis of buckling, free vibration and flexure of clamped laminated composite plates in variable thermal environment. Advances in Mechanical Engineering (2020) 1151-1161.

8. Jeyaraj P.: Buckling and free vibration behaviour of an isotropic plate under non-uniform thermal load. International Journal of Structural Stability and Dynamics 13 (3) (2013) 1250071.

9. Sai Ram K.S, Sinha P.K.: Hygrothermal effects on the bending characteristics of laminated composite plate. International Journal of Computers and Structures 40 (4) (1991) 1009-1015.

10. Twinkle C.M., Pitchaimani J., Rajamohan V.: Free vibration modes of rectangular plate under nonuniform heating: An experimental investigation. Structures 28 (2020) 1802-1817.

11. Yang X., Fei Q., Wu S., Li Y.: Thermal buckling and dynamic characteristics of composite plates under pressure load, Journal of Mechanical Science and Technology 34 (8) (2020) 3117-3125.

12. Abidaa M., Gehringan F., Marsb J., Viveta A., Dammakb F., Haddarc M.: Hygro-mechanical coupling and multiscale swelling coefficients assessment of flax yarns and flax/epoxy composites. Composites Part A 136 (2020) 105914.

13. Gayen D., Roy T.: Hygro-thermal effects on stress analysis of tapered laminated composite Beam. International Journal of Composite Materials 3 (3) (2013) 46-55.

14. Biswal M., Sahu S.K., Asha A.V., Namita Nanda.: Hygro-thermal effects on buckling of composite shell-experimental and FEM results. Steel and Composite Structures 22 (6) (2016) 1445-1463.

15. Patel B.P., Ganapathi M, Makhecha D.P.: Hygro-thermal effects on the structural behaviour of thick composite laminates using higher-order theory. Composite Structures 56 (2002) 25-34.

16. Sahin O.S.: Thermal buckling of hybrid angle-ply laminated composite plates with a hole. Composites Science and Technology 65 (2005) 1780-1790.

17. Chikh A., Tounsi A., Hebali H., Mahmoud S.R.: Thermal buckling analysis of cross-ply laminated plates using a simplified HSDT. Smart Structures and Systems 19 (3) (2017) 289-297.

18. Chandrashekhara K.: Thermal buckling of laminated plate using a shear flexible finite element. Finite Elements in Analysis and Design 12 (1992) 51-61.

19. Sun L.X, Hsu T.R.: Thermal buckling of laminated composite plates with transverse shear deformation. Computers \& Structures 36 (5) (1990) 883-9.

20. Chockalingam S., Mathew T.C., Singh G., Rao G.V.: Critical temperatures of hybrid laminates using finite elements. Computers \& Structures 43 (5) (1992) 995-8.

21. Thangaratnam K.R., Ramachandran J.: Thermal buckling of composite laminated plates. Computers \& Structures 32 (1989) 1117-24.

22. Chen L.W., Lin P.D., Chen L.Y.: Thermal buckling behaviour of thick composite laminated plates under non-uniform temperature distribution. Computers \& Structures 41 (1991) 637-45.

23. Kallannavar V., Kumaran B., Kattimani S.C.: Effect of temperature and moisture on free vibration characteristics of skew laminated hybrid composite and sandwich plates. Thin-Walled Structures 157 (2020) 107113. 
24. Thai C.H., Kulasegaram S., Tran L.V., Nguyen-Xuan H.: Generalized shear deformation theory for functionally graded isotropic and sandwich plates based on isogeometric approach. Computers \& Structures 141 (2014) 94-112.

25. Nguyen T.N, Thai C.H., Nguyen-Xuan H.: On the general framework of high order shear deformation theories for laminated composite plate structures: A novel unified approach. International Journal of Mechanical Sciences 110 (2016) 242-255.

26. Nguyen T.N., Ngo T.D., Nguyen-Xuan H.: A novel three-variable shear deformation plate formulation: Theory and Isogeometric implementation. Computer Methods in Applied Mechanics and Engineering 326 (2017) 376-401.

27. Bathe K.J.: 2014. Finite Elements Procedures. Second Edi. Prentice Hall, Pearson Education, Inc.

28. Maharudra, B. Arya, Rajanna T.: Effect of ply-orientation and boundary conditions on the vibrational characteristics of laminated composite plates using HOSDT, Materials Today: Proceedings. 20 (2020) 134-139.

29. Rajanna T., Banerjee S., Desai Y.M., Prabhakara D. L.: Effects of partial edge loading and fibre configuration on vibration and buckling characteristics of stiffened composite plates. Latin American Journal of Solids and Structures 13 (2016) 854-879.

30. Muddappa P.P.Y., Rajanna T., Giridhara G.: Effects of different inter-laminar hybridization and localized edge loads on the vibration and buckling behaviour of fiber metal composite laminates. Composites Part C 4 (2021) 100084.

31. Subash Chandra K.S., Rajanna T., Venkata Rao K.: A parametric study on the effect of elliptical cutouts for buckling behaviour of composite plates under non-uniform edge loads. Latin American Journal of Solids and Structures 17 (2020) 1-15. 